\title{
Elementos de un modelo de aprendizaje a distancia en tiempos de COVID-19
}

Luis Octavio Alpizar Garrido ${ }^{a}$

${ }^{a}$ Colegio Indoamericano, S.C., Tlalnepantla, Estado de México, México. luisoctavioalpizar@outlook.com

\section{\$EWWFW}

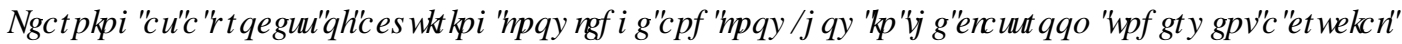

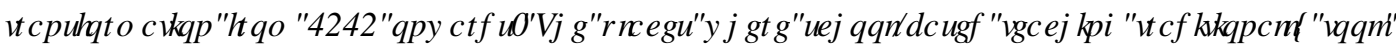

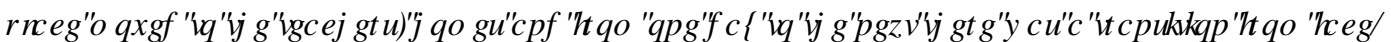

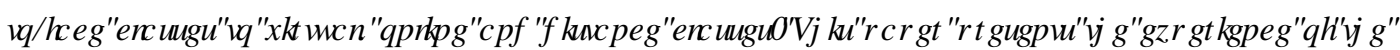

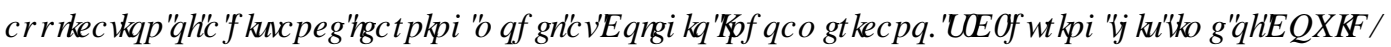

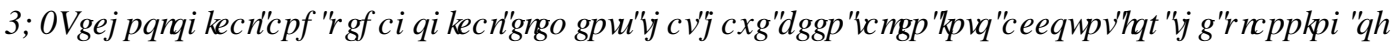

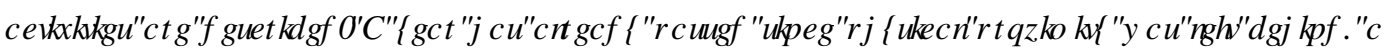

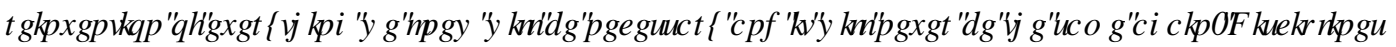

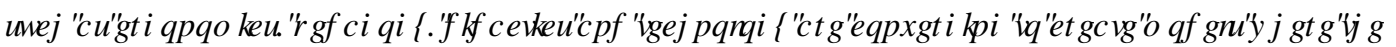

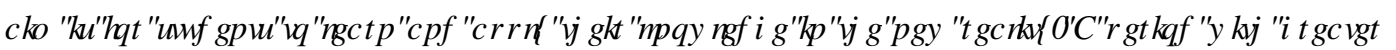

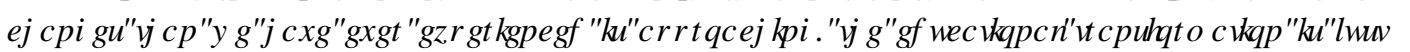
EHIQQDТ]

\section{. HZRLVII IUKDOCDQQQJ}

\section{HXP HQ}

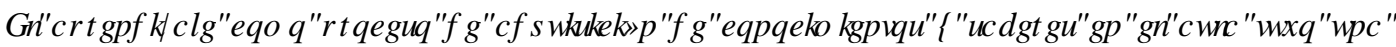

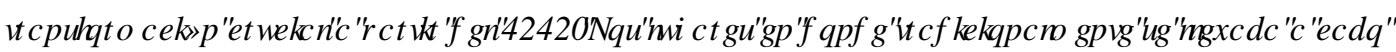

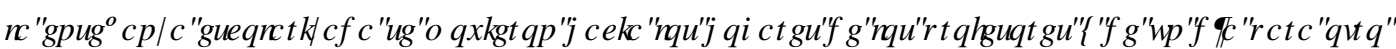

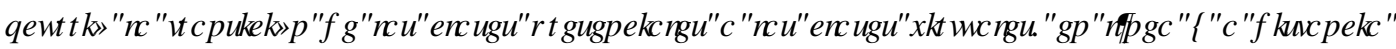

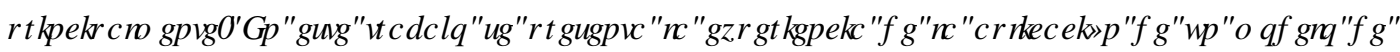

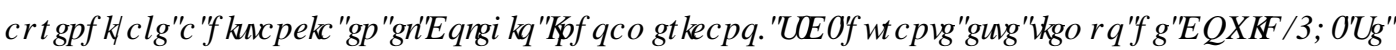

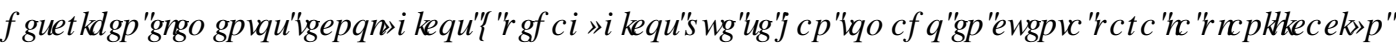

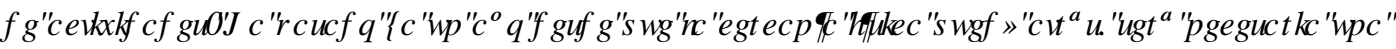

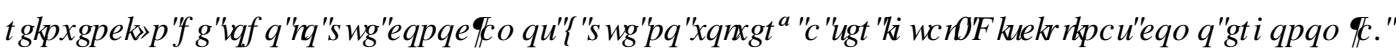

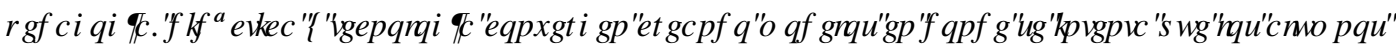

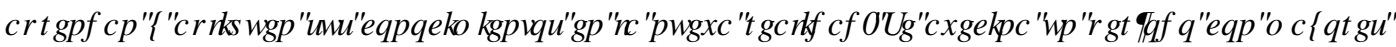

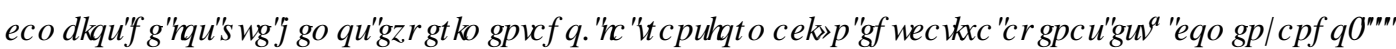

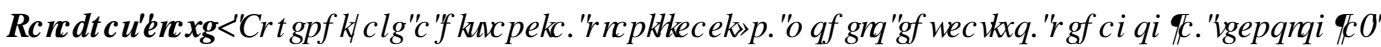




\section{Introducción}

De forma completamente inesperada hizo su aparición en el mundo el COVID-19. La Organización Mundial de la Salud (OMS) informaba en enero del 2020 que estábamos primero ante una emergencia de salud pública de emergencia internacional, para dos meses más tarde, ya en marzo, declararla como pandemia (OPS, 2020). A partir de ese momento todo cambió, gran parte de la población mundial se confino a sus hogares.

En la esfera de la educación, tuvo lugar al cierre masivo de las actividades presenciales de instituciones educativas en más de 190 países con el fin de evitar la propagación del virus y mitigar su impacto. (Informe COVID-19 CEPAL-UNESCO, 2020). Las instituciones educativas tuvieron que comenzar la educación a distancia con los recursos que tenían a la mano (los que los tenían).

Responsables académicos, docentes, estudiantes y familias emprendieron acciones de manera urgente hacia una nueva faceta: la imposible presencialidad. (Porlán, 2020). El coronavirus trajo consigo la adecuación de técnicas y herramientas innovadoras para el aprendizaje; de esta manera, la educación a distancia, pensada para aquellos que disponían de poco tiempo para asistir a las instituciones educativas, se convirtió para quienes asistían de forma presencial, de un viernes a un lunes en un escenario nuevo e inexplorado.

Ante este estado, poco a poco empresas de tecnología y telecomunicaciones han incrementado de forma considerable sus servicios, programas, software y hardware. (García, 2021). Esto sirvió de apoyo para quienes realizan actividades laborales desde casa (Home Office), así como también, en el ámbito educativo, para mantener tanto la conexión como la comunicación entre profesores y estudiantes.

Las clases no se detuvieron. Cada nivel educativo encontró la forma de dar continuidad al proceso de enseñanza aprendizaje a través de distintos medios. Los niveles educativos básicos lo resolvieron dando clases por medio de programas de televisión, en el caso de los niveles más altos, educación media superior y superior, su salvación fue el uso de aplicaciones vía Internet.

El Colegio Indoamericano, S.C., una institución de educación media superior ubicado en el municipio de Tlalnepantla, Estado de México, México. Con más de 45 años de experiencia en el mercado, el colegio había venido impartiendo educación preparatoria en modalidad presencial. El ciclo escolar 2019-2020 finalizó para un poco más de 1,000 estudiantes recibiendo clases en forma remota. El escenario prevalece en esa modalidad durante el ciclo escolar 2020-2021, con un breve camino recorrido, pero con grandes experiencias adquiridas.

Muchas universidades en todo el mundo continúan sin adaptarse a las restricciones sanitarias para dar educación de manera remota, su prioridad ha sido ajustarse a la crisis, garantizar pequeños éxitos permanentes de aprendizaje y ser empáticos con la realidad socioemocional de sus comunidades. (Pardo y Cobo, 2020, p. 5)

Estamos ante un hecho sin precedentes en la historia, aún con el avance tan grande en la ciencia y la tecnología, la educación no se ha transformado en la herramienta de conocimiento acorde a los estándares y exigencias que la sociedad actual requiere. El modelo educativo sigue anclado al pasado, con un esquema que tiene al docente como protagonista, la memorización de la información y la evaluación por vía de pruebas estandarizadas. En algunos lugares ya se han atrevido a implementar alternativas de educación, ya sea a distancia, en línea, síncrona, etc. Dichas evidencias permiten albergar esperanzas para que ocurra una migración irreversible: aprendizaje fuera de las cuatro paredes del aula, emplear recursos más allá del docente como proveedor de información, empoderamiento del estudiante, atemporalidad del aprendizaje y expansión de las posibilidades del conocimiento. (Pinzón, 2021, p. 15). 
El presente trabajo pone de manifiesto no solo lo que se ha hecho, sino diversos resultados obtenidos a partir del modelo de aprendizaje en línea implementado en el Colegio Indoamericano, S.C. Se mencionan actividades que asignan los profesores y cómo a través de ellas propician el desarrollo de habilidades por parte de los estudiantes. Finalmente, se proponen instrumentos los cuales pueden disponer en alguna institución de educación media superior e incluso superior, diseñando e implementando un modelo de aprendizaje a distancia.

\section{Objetivos}

El objetivo de principal de este trabajo es exponer la forma en que se ha llevado a la práctica el modelo de aprendizaje a distancia en el Colegio Indoamericano, S.C.

Los objetivos específicos serían:

- Mostrar los elementos relevantes del modelo de aprendizaje a distancia que se ha utilizado.

- Mencionar recursos y herramientas que han servido para definir las líneas de acción en el diseño de una propuesta metodológica virtual para el trabajo académico en el Colegio Indoamericano, S.C. durante y después de la pandemia.

- Evaluar la satisfacción de profesores y estudiantes ante esta nueva modalidad de enseñanza aprendizaje.

La optimización de este proyecto de innovación escolar tiene como intención proporcionar a los estudiantes la mejor experiencia de aprendizaje con una modalidad adaptada a estos tiempos.

\section{Desarrollo de la innovación}

Para la realización de esta investigación se utilizó el método documental y el enfoque cualitativo. En la obtención de datos por medio la aplicación de la encuesta se empleó el enfoque cuantitativo.

El proyecto de innovación en el Colegio Indoamericano, S.C. inició a partir del ciclo escolar 2018-2019. Desde esa fecha, la comunidad académica se viene preparando para que la formación pedagógica esté mediada por la tecnología. A continuación, se enlistan los elementos clave que intervienen en el modelo de educación a distancia vigente en el colegio:

\section{Enseñanza-aprendizaje.}

Para entender el sistema actual de enseñanza aprendizaje aludiremos un poco a la historia y sobre todo a la filosofía. Respecto al método que prevalece gran parte de las escuelas, Márquez (2018) se refiere a los inicios de las universidades, en particular al método escolástico y a sus tres fases más conocidas: Lectio, Quaestio y Disputatio. A la lectio como la lectura de textos dependiendo de la materia que se enseña, la quaestio representa la pregunta que da lugar al análisis del tema y disputatio sería el debate, la discusión entendida de la mejor manera. Este proceso se venía practicando con regularidad de manera presencial en las aulas, con la pandemia, ahora se hace gracias a una pantalla como interfaz, pero sigue siendo casi lo mismo.

En su charla "Presencia en el aula virtual", Aldana nos habla de cómo la situación actual nos obligó a una continuidad pedagógica desde casa. Es a través de lo que él denomina rectángulo aula que los estudiantes aprenden y los profesores enseñan, de tiempo completo a través de la pantalla del computador o del dispositivo móvil. (Aldana, 2020, 16m41s). 
Para (Lion, 2020) las condiciones están dadas para diseñar propuestas educativas de relevancia, contextualizadas y con sentido pedagógico. La misma autora menciona que se trata de tomar decisiones acerca de: lo que vale la pena enseñar con mediación tecnológica (sincrónica y asincrónica), determinar cuáles contenidos son vitales y cuales periféricos; cómo situar actividades para que tengan sentido en relación con las trayectorias e intereses de nuestros/as estudiantes y no solo porque "son temas que hay que enseñar"; la mejor forma para conjugar lo cognitivo con lo emocional; cómo entramar lo moderno con lo antiguo y cómo abordar la diversidad que siempre ha existido y existirá en el ámbito escolar.

\section{Diseño instruccional.}

Para Ortega (2020, p. 2), el desarrollo de contenidos educativos en cualquier modalidad exige una didáctica adecuada tanto al método de enseñanza del docente, como al estilo de aprendizaje del estudiante; el profesional de la educación debe incluir canales eficientes de comunicación para que los conocimientos permeen adecuadamente.

El modelo de diseño instruccional que aplica el Colegio Indoamericano ha sido comunicado a miembros de la comunidad académica, estudiantes y padres de familia. La enseñanza virtual y a distancia cumple una serie de lineamientos muy precisos: puntualidad en las clases, sesiones con fases plenamente diferenciadas (inicio, desarrollo y cierre), explicaciones claras, desarrollo natural de actividades y algo muy importante, retroalimentación a las dudas o preguntas de los estudiantes.

Actividades docentes sustantivas como la evaluación, se ajustó a la modalidad en línea dando una mayor ponderación a la evaluación formativa o continua, al tiempo que disminuyen el porcentaje asignado a la evaluación sumativa o lo que conocemos comúnmente como exámenes.

Independientemente del período en que nos encontremos, en el colegio se integra nuestra metodología de enseñanza con el uso educativo de la tecnología para apoyar el aprendizaje de los alumnos, durante su vida escolar los alumnos se capacitan para el uso ético y responsable de las Tecnologías de Información y Comunicación (TIC), se fortalece creativamente el desarrollo de habilidades de pensamiento crítico que les permitan aprender a aprender, aprender para la vida y hacerlo de manera permanente (Portal Institucional del Colegio Indoamericano, S.C.).

\section{Profesores.}

Al igual que todos, los profesores se vieron sorprendidos y de repente ya no impartirían más clases presenciales, ahora serían en la virtualidad y a la distancia. En el caso específico de la Facultad Académica del Colegio Indoamericano, las continuas sesiones de capacitación de ciclos escolares previos dieron a los docentes las herramientas básicas en las áreas socioemocional, pedagógica y tecnológica que los prepararon para este nuevo panorama. Generación de nuevos programas de estudio, adaptación de contenido de materias existentes e inversión en tiempo para aprender técnicas y estrategias digitales nuevas. Estos son solo unos cuantos ejemplos de cómo se construía un nuevo entorno educativo que, si bien no resolvió el problema, permitió afrontarlo con cierta dosis inicial de tranquilidad y confianza.

En la opinión de Rivas (2020), no serán los docentes expertos en tecnología los mejor posicionados para rehacer el sistema, serán los conocedores (y los apasionados) de la didáctica, los que buscan reinventar la enseñanza con sentido y criterio en las condiciones que la realidad les presenta. Es claro que la tecnología ayudará, pero mucho más importante será la adaptación desde la mirada pedagógica. La ruta de la tecnología digital que anticipa se convertirá en un gran diferenciador de las posibilidades de recuperar la experiencia educativa en la ausencia de las aulas.

Desde el inició el confinamiento, la Facultad Académica del Colegio Indoamericano, S.C. imparte todas sus clases de manera síncrona en días y horas predeterminadas. 
La Figura 1 muestra la clase de la materia de biología impartida en modalidad virtual por parte de una de sus profesoras titulares.

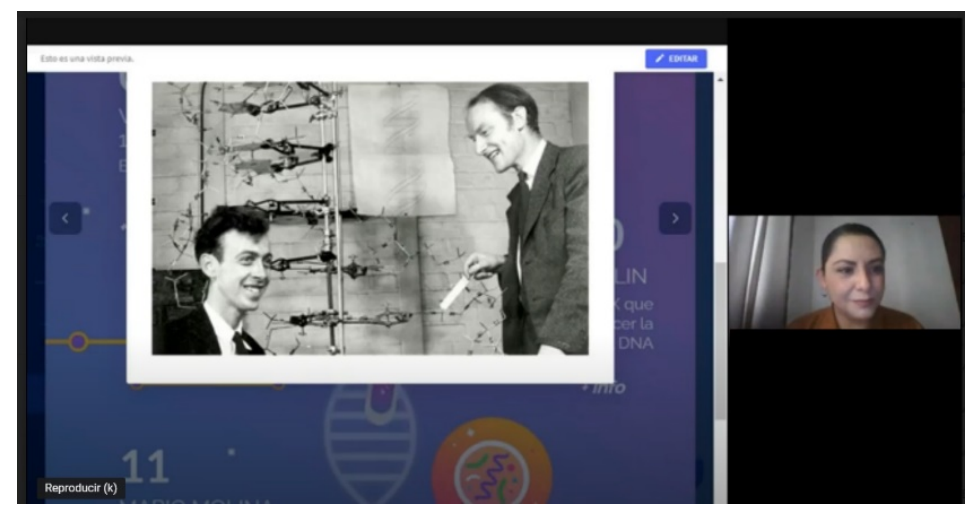

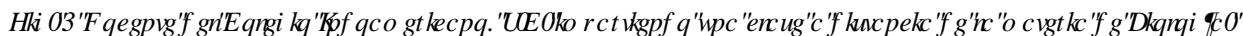

Es primordial que las universidades y centros educativos lleven a cabo programas de actualización y formación para todos los docentes, que les permitan acceder a herramientas y recursos de vanguardia que apliquen en sus clases. Importante también hacerles llegar conceptos y corrientes nuevas como las llamadas pedagogías emergentes, promover con mayor frecuencia la aplicación de estrategias como el design thinking, la gamificación, el flipped classroom y otras iniciativas provenientes de muchos lugares del mundo (Aparicio, 2021).

\section{Estudiantes.}

Es menester, además, concebir al estudiante como elemento central en todo este proceso de reinvención. Reconocer el protagonismo que merecen e invitarlos continuamente a participar, proponer, intentar, construir y atreverse a luchar por sus objetivos. Aun estando la pantalla como separación entre el docente y el estudiante, en cada lugar en el que se recibe educación se puede transformar el entorno, aprender cosas nuevas, aplicar conocimientos y emprender innovadores proyectos.

El Colegio Indoamericano, S.C. se esfuerza por informar oportunamente a los estudiantes. Se utilizan diversos canales para lograr esto y entre éstos se encuentra el blog institucional (Colegio Indoamericano, 2020). En estos espacios el estudiante encuentra información de actualidad respecto a diversos temas tales como: vida escolar, técnicas de estudio, riesgos de la adolescencia, entre otros.

Un elemento de especial atención hacia la asistencia a clases por parte de los estudiantes es el horario de clases. La dirección académica ha creado un horario acondicionado a estos tiempos. La Figura 2 muestra el horario escolar que tienen los estudiantes durante el actual ciclo escolar.

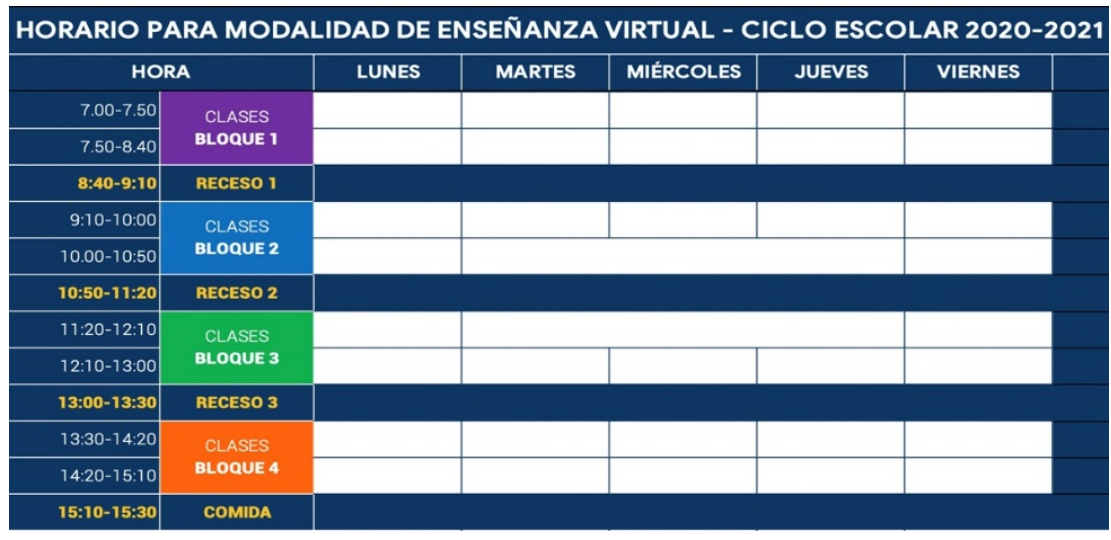

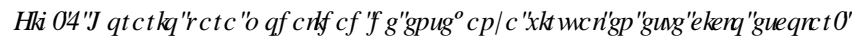


La atención hacia la preparación de los estudiantes es total. Entre los objetivos de los docentes para con ellos están: mayor acercamiento a la tecnología digital con énfasis desde cada una de sus asignaturas, fortalecer sus hábitos de estudio, tener proximidad con el estudiante y empatía con su edad de desarrollo, promover su autonomía y autorregulación, elevar su nivel de pensamiento crítico y de investigación.

\section{Plataforma y herramientas tecnológicas.}

La plataforma base de trabajo es la G Suite de Google for Education. A través de ella se gestionan clases, tareas, participaciones virtuales y proyectos de las diferentes materias. Les ha permitido a los alumnos tener experiencias de trabajo escolar de forma dinámica y hasta divertida. Gracias a la infraestructura con la que cuenta el Colegio Indoamericano, S.C., estas aplicaciones en su conjunto le permiten mantener un ambiente proactivo en donde prevalece el ánimo e interés por aprender.

El proceso de comunicación es vital en cualquier organización y no es la excepción en instituciones educativas. Para la comunicación interna y formal usamos la plataforma Colaboranet, como plataforma educativa Google Classroom. La Figura 3 muestra ambas aplicaciones que mantienen viva la comunicación con las personas que integran la comunidad escolar.

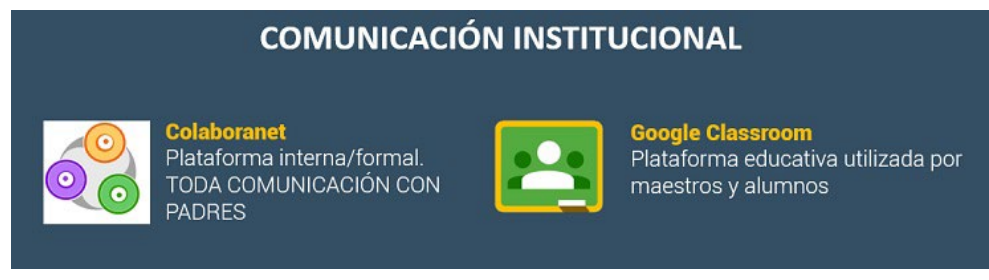

) $I J W \square$ W SQFDFIRQHUGHFRP XQLFFIY QIQMUXFIRQDO

Los elementos de carácter técnico que deben tener en cuenta para las clases a distancia, tanto profesores como estudiantes son: velocidad de Internet confiable, micrófono, sonido y cámara de video, espacio acondicionado para tomar la clase, sistema de gestión de aprendizaje (de sus siglas en inglés, LMS, Learning Management System) que en nuestro caso es Google Classroom y la plataforma de videollamada, Google Meet.

Diversos autores han documentado sus mejores propuestas ante un mar de información, es el caso de la Cátedra digital, un concepto en donde los autores proponen un modelo que tiene como base, por un lado, un ambiente virtual de aprendizaje (AVA), y por el otro, una plataforma de videollamada. Sobre ellos ocurren actividades, se realizan conexiones y se comparten recursos (García, 2021). Así como ellos, en el caso de la facultad académica del Colegio Indoamericano, S.C., utilizamos la infraestructura tecnológica basada en Google Workspace for Education, tomando a Google Classroom como AVA, a Google Meet como plataforma de videoconferencia y Google Calendar como administrador de tiempos escolares.

El tema de la o las plataformas tecnológicas de trabajo debe ser realizada con total compromiso y seriedad, se deben analizar cuidadosamente las alternativas y se debe elegir lo más conveniente para la comunidad, no únicamente docentes y estudiantes, sino considerando a todas las partes interesadas en el proceso educativo.

La variedad de opciones a las que se puede acceder en este momento es prácticamente ilimitada, existen posibilidades gratuitas, de pago y con suscripción. El número de aplicaciones utilizadas por los docentes ha crecido de manera exponencial, y las utilizan ya sea para grabar videos, elaborar presentaciones electrónicas, crear libros digitales, formular un examen en línea, crear un canal de podcast, etc. La oferta seguirá creciendo, seguirán aumentando las posibilidades de agregar variedad y calidad a los contenidos educativos. 


\section{Espacios y recursos en la nueva realidad.}

Uno de los cuestionamientos permanentes acerca de la educación es ¿por qué se conservan elementos de organización escolar del siglo pasado en una época en la que muchas otras áreas han tenido cambios progresivos? El SARS-CoV-2, otro nombre por el que se conoce al coronavirus ha puesto aún más en la mira esta cuestión y se tiene la esperanza de que impulse una franca innovación educativa. Y es que innovar no es solo introducir recursos tecnológicos o metodologías novedosas al aula. Para (Mosquera, 2019) un concepto clave es adaptación, a las circunstancias que tenemos en el contexto de enseñanza aprendizaje en el que nos encontramos. Innovar es un proceso constante en el que todos los actores debemos estar conscientes de que requerimos una continua formación y también tener una mente abierta para adoptar los cambios.

En una época en donde el aprendizaje a distancia no reconoce límites o barreras físicas, vuelve a ponerse de moda un término que hace un par de año se comenzaba a escuchar, el término hiperaula. Es un concepto que considera la reconfiguración de aulas y de espacios de aprendizaje, Fernández (2019) destaca la existencia de iniciativas escolares que intentan romper con la vieja fórmula de un docente, un aula, una asignatura y una hora, para trasladarse a espacios y tiempos más amplios y flexibles, con nuevas variables, grupos más numerosos, dos docentes o más y vasto uso de tecnología digital.

Para Walsh (2020) existe la necesidad de espacios de aprendizaje innovadores que ayuden a los estudiantes a construir conocimiento. Justifica su pensamiento exponiendo cinco razones: Redefinición del concepto de espacio, mejoramiento de la colaboración y la flexibilidad, promoción de agrupamiento y gestión del tiempo, fomento del aprendizaje individualizado y creación de oportunidades fuera de la escuela.

Diversos autores han descrito hipotéticos escenarios para la etapa post-COVID-19, tal es el caso de Pardo y Cobo (2020: 34-39) quienes plantean el rediseño obligado de los cursos presenciales hacia la enseñanza remota de emergencia. El primer escenario lo denominan: Remoto urgente no adaptado, que básicamente se centra en seguir el proceso de enseñanza aprendizaje previsto, cumplir en la crisis y que el estudiante apruebe con una calificación; el segundo: Remoto urgente adaptado, la meta es similar a la del primer escenario con variación en el método, en este caso, se reduce el margen de improvisación pero el docente sigue teniendo el control del aprendizaje de sus estudiantes; el tercer escenario: Remoto aspiracional, aquí el rol del profesor cambia al de diseñador de experiencias de aprendizaje, ya tiene un mayor control de los tiempos de ejecución de tareas y el trabajo autogestionado, factor que el estudiante aprovecha para mejorar su aprovechamiento y rendimiento; por último un cuarto escenario: Híbrido aspiracional, con todas las ventajas de los escenarios anteriores, mezcla permanente y constante de los ambientes tanto remotos como físicos o presenciales, en este nivel el docente ya lleva a cabo la integración definitiva de experiencias remotas exitosas.

De acuerdo con Arcos (2020) la educación enfrentará en los próximos años varias revoluciones: tecnológica, pedagógica y social. Seremos testigos de una incremental influencia de la educación expandida, el autoaprendizaje, las llamadas competencias blandas y más nociones que superan el recinto escolar y extienden los espacios y tiempos de aprendizaje.

\section{Resultados}

El instrumento diseñado para identificar la percepción de los estudiantes del Colegio Indoamericano, S.C. respecto a los efectos del COVID-19 sobre el proceso educativo, se validó mediante juicio de expertos. 
Se aplicó una encuesta para identificar la experiencia ante esta nueva modalidad de enseñanza-aprendizaje. Esto ocurrió en el mes de junio de 2020 y fue contestada por el $70 \%$ de la población estudiantil del colegio. Cabe recordar que al momento de realizar la encuesta tenían cerca de cuatro meses de haber comenzado las clases en educación a distancia.

Por su representatividad y por razones de extensión de este escrito, se exponen seis de los ítems y sus resultados.

Al preguntar a los alumnos si consideraban que el colegio estaba preparado para continuar con clases en línea, un porcentaje menor al 83\% consideró que sí, como se observa en la Figura 4.

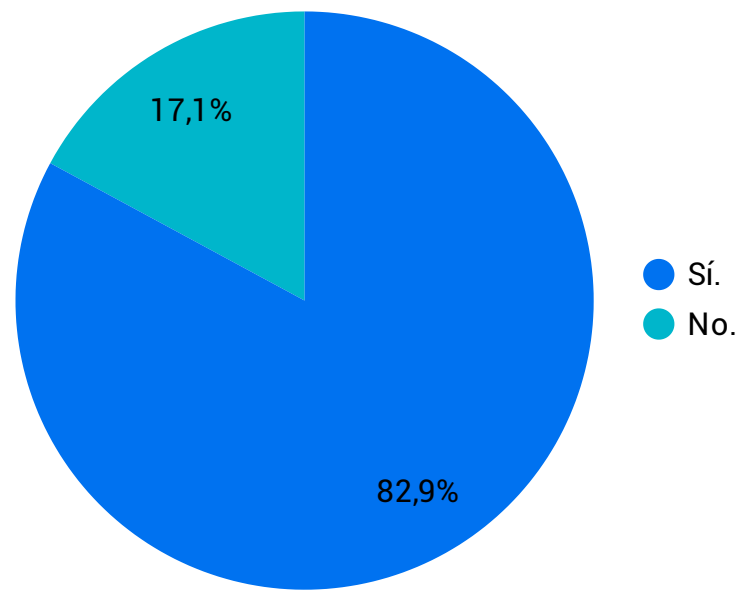

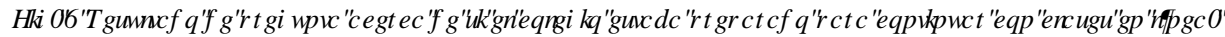

En la Figura 5 se aprecian los resultados a la pregunta ¿Consideras que tus profesores estuvieron preparados para enfrentar adecuadamente el reto de la enseñanza a distancia? En este caso, un porcentaje cercano al 95\% confirmó la idea de que los profesores estaban adecuadamente preparados para este reto, que las horas de formación previa estaban rindiendo frutos, que se tenía que continuar por ese camino y mejorar aún.

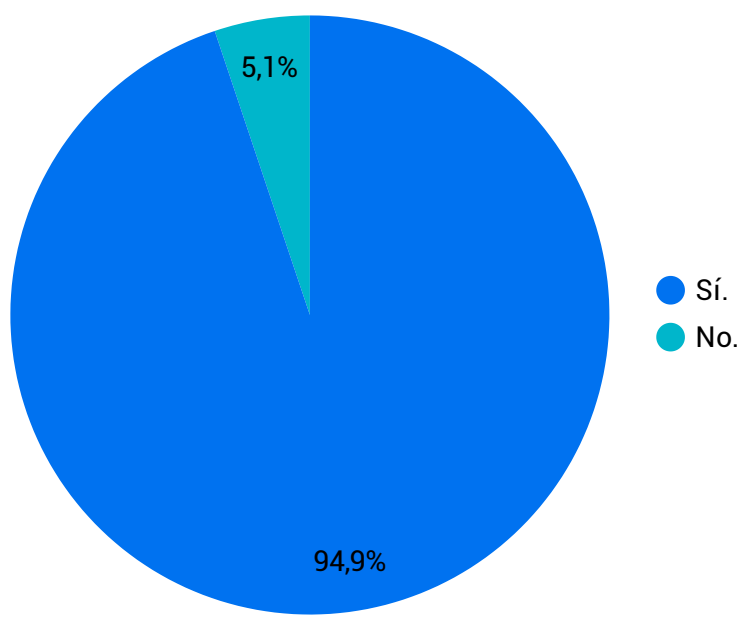


La Figura 6 muestra el resultado de la respuesta que se obtuvo al preguntar a los estudiantes si se había sentido cercano y vinculado a sus maestros durante el período de enseñanza a distancia.

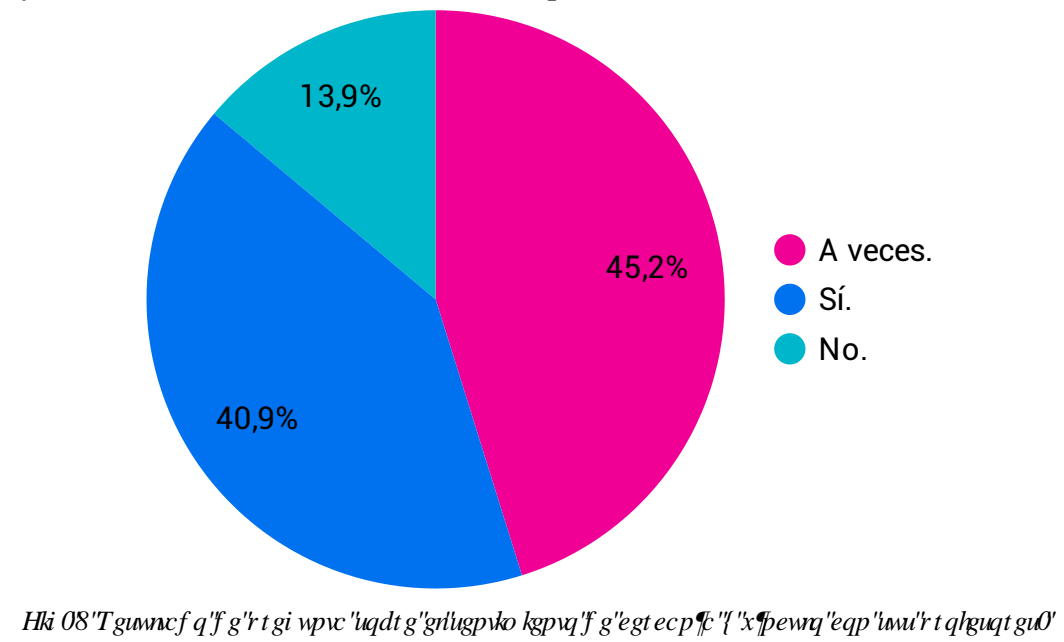

La respuesta a la pregunta: De acuerdo con tu experiencia, ¿cómo te gustaría continuar tu educación? Se muestra en la Figura 7. Se entiende por qué había transcurrido relativamente poco tiempo de que los estudiantes habían dejado de tener clases presenciales.

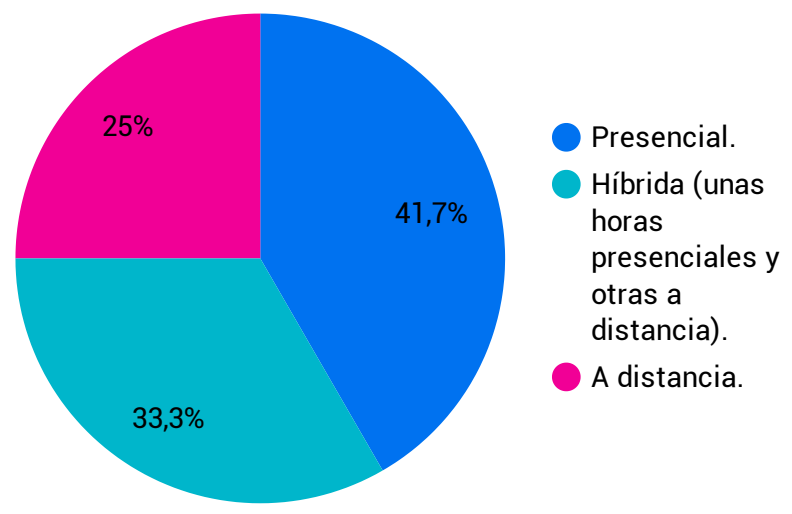

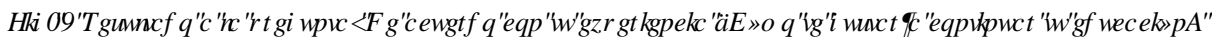

En la Figura 8 se aprecia el resultado a la pregunta: ¿en qué tipo de modalidad consideras que obtienes mejores resultados de aprendizaje? El comportamiento en cierto modo extraño, ya que los estudiantes estaban obteniendo mejores notas en modalidad en línea con respecto a la modalidad presencial.

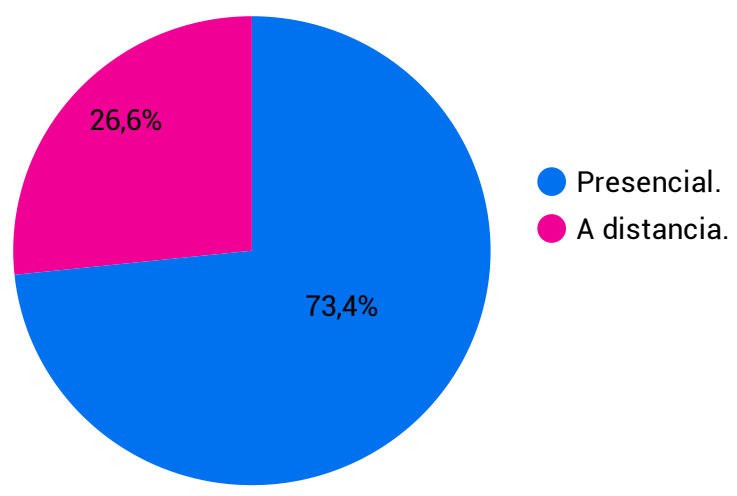

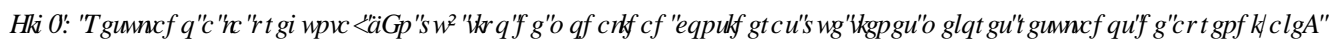

(cc) BY-NC-ND 2021, Universitat Politècnica de València

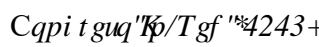


Respecto a la pregunta ¿conoces para qué sirven cada una de las aplicaciones que integran la G Suite?, en la Figura 9 se ve fragmentado el conocimiento, esto puede deberse a que la encuesta fue respondida por alumnos de los tres grados. Es posible que el nivel de seguridad en el manejo de las herramientas no les permitió responder que conocían su utilidad. La asiduidad en el uso de las aplicaciones, no sólo de la G Suite sino en general de herramientas digitales se incrementa de una forma notable durante su estancia en el Colegio Indoamericano, S.C. y eso lo expresan alumnos y exalumnos.

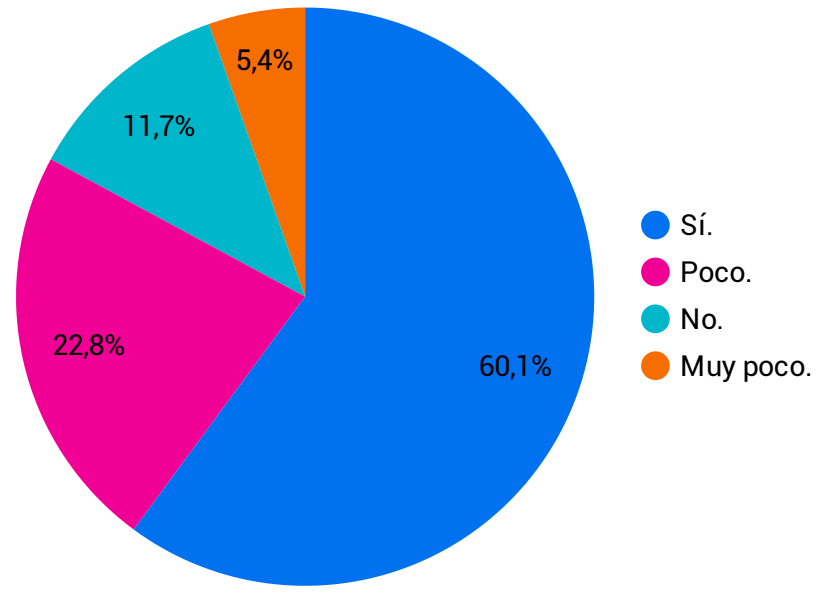

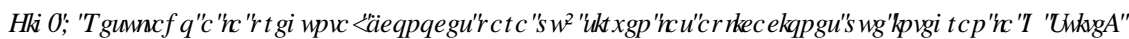

En la Figura 10 se observa que afortunadamente un porcentaje elevado de alumnos tiene la posibilidad de conectarse a sus clases con algún dispositivo electrónico, ya sea computadora portátil o de escritorio, tableta o teléfono inteligente.

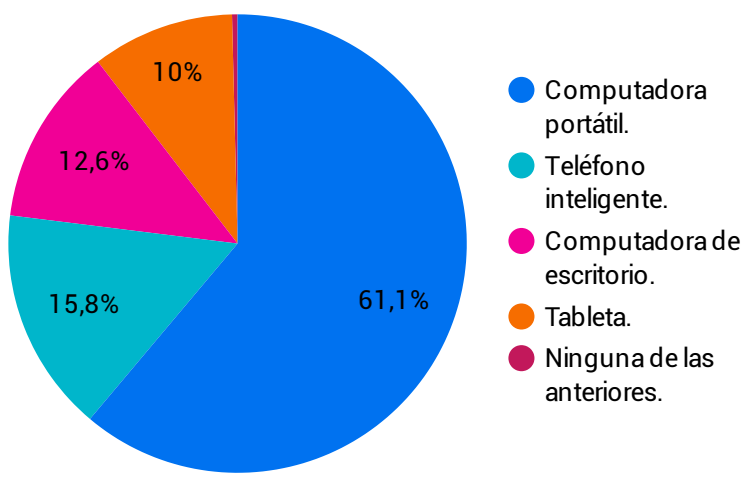

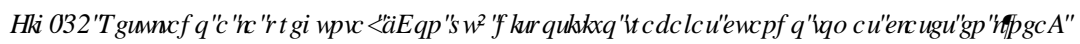

Una recomendación de elemento a incluir en el modelo de aprendizaje a distancia es un equipo de personas que se encargue de la analítica de datos, resulta bastante útil y conveniente ya que permite tener el pulso a un momento determinado de lo que sucede en la universidad o colegio. Entre la información que se puede analizar periódicamente está: asistencia de alumnos a clases virtuales, porcentaje de ausencias, asistencia de docentes a sus sesiones de clases, materias en las que hay mayor índice de reprobación, estudiantes con problemas de conexión o de tipo técnico que les impida conectarse, etc.

El personal del Departamento de Tecnología trabaja para acercar la mayor cantidad de aplicaciones que les sirvan a los estudiantes para mejorar sus habilidades. Un estudiante del colegio tiene acceso completo y soporte de las siguientes plataformas y herramientas: Microsoft Office, la Suite de Adobe, hardware y software de Apple, y por supuesto la Suite completa de Google. 
A continuación, se mencionan algunos de los resultados que se han obtenido a lo largo de los últimos doce meses sobre el comportamiento de distintos rubros:

- Creación y publicación a la comunidad escolar del Reglamento Institucional para clases virtuales.

- Disponibilidad de biblioteca digital para consulta bibliográfica y realización de trabajos de investigación.

- Grabación por parte de los profesores del 100\% de las sesiones de Google Meet y de clases de Google Classroom en Google Drive.

- Registro en una bitácora digital del avance programático diario.

- Registro de asistencia de alumnos de todas las sesiones que se imparten.

- Servicio de soporte técnico vía correo electrónico, chat y por teléfono.

- Sesiones virtuales cada semana, tanto de acompañamiento institucional como de relajamiento para el personal del colegio.

- El porcentaje promedio de conexión de estudiantes por grado a sesiones de clase sincrónicas se mantiene arriba del 95\%.

- Trabajo diferenciado con estudiantes que por razones técnicas no tengan posibilidad de conectarse a sus clases.

- El porcentaje de clases en Google Classroom es del 100\%.

- El porcentaje de profesores preparados y en continua capacitación es del 100\%.

\section{Conclusiones}

Estamos a poco más de un año de permanecer en confinamiento y de haber comenzado sesiones de clase a distancia. En todo este tiempo se han tenido muchas experiencias y se pueden rescatar bastantes aprendizajes. Se han compartido los elementos, instrumentos y estrategias que se han aplicado, así como los resultados obtenidos con ellos. Con una mirada retrospectiva, es posible decir que, gracias a las acciones implementadas, hoy por hoy los docentes del Colegio Indoamericano, S.C. han brindado a sus estudiantes nuevas posibilidades, les han enseñado a adquirir habilidades que antes no tenían.

Siempre un momento de crisis nos debe alentar a pensar alternativamente, a vislumbrar nuevos horizontes. En definitiva, es una oportunidad para identificar aquello que nos han sorprendido positivamente, para rescatar los ejercicios que una vez que logramos tener, reconocimos como valiosos y útiles. Revalorizar no significa ignorar aquellos hechos que nos incomodaron o que no salieron como esperábamos. Apreciar estos matices permitirá hacer conciencia, reflexionar y avanzar con más ánimo.

El tiempo transcurrido nos permite adaptarnos a la nueva situación en que vivimos. La adecuación correcta de las condiciones para impartir la educación a distancia, son el punto inicial para el desarrollo fluido y armonioso de las sesiones de clase. Los docentes, estudiantes y familias son y seguirán siendo los agentes principales de cambio en este proceso.

Se trabaja día con día por construir un modelo propio de educación a distancia que beneficie el aprendizaje por parte de los estudiantes del colegio. Su estructura es un rompecabezas de muchas piezas que se va armando de forma progresiva. Nos falta mucho camino por recorrer, no hay indicios de cuál será el camino a seguir, ¿será en modalidad presencial?, ¿continuará el aprendizaje en línea?, ¿se optará por la modalidad híbrida? Sea cual sea la respuesta, las instituciones tienen que innovar e invertir en sus recursos tanto materiales como humanos para responder adecuadamente a las circunstancias actuales.

Seguiremos poniendo el mejor empeño en nuestras acciones para destacar en todo momento.

(c)) BY-NC-ND 2021, Universitat Politècnica de València

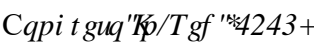




\section{Referencias}

ALDANA, H. (2020). "Presencia en el aula virtual | TEDxPuraVidaSalon". $<R X W E H<$ https://youtu.be/oi4vo0YoEKM $>$ [Consulta: 22 de mayo de 2021]

APARICIO GOMEZ, O. Y. (2021). "Pedagogías emergentes en ambientes virtuales de aprendizaje" en 5HILW , QUAQDFIRQDO GH 3 HDDJUtDD H , QQRVDFIyQ ( GXFDUMDD 1. 11-36. <http://dx.doi.org/10.51660/ripie.v1i1.25> [Consulta: 08 de marzo de 2021]

ARCOS, A. (2020). "Las nueve tendencias educativas que marcarán este 2020" en 0 DJLMALRD( VSHFLOS QXDUR

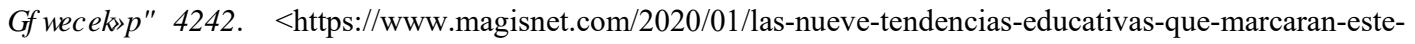
2020/> [Consulta: 25 de marzo de 2021]

COLEGIO INDOAMERICANO (2021). “iPara qué me sirven las herramientas de la G Suite?” en Blog del Colegio Indoamericano, S.C., 12 de marzo. <https://blog.indo.edu.mx/para-que-sirven-herramientas-de-g-suite> [Consulta: 18 de marzo de 2021]

FERNANDEZ ENGUITA, M. (2019). "Hiperaulas: así es la escuela que desbancará al colegio tradicional" en 7KH $\& R Q Y H W D R Q<$ https://theconversation.com/hiperaulas-asi-es-la-escuela-que-desbancara-al-colegio-tradicional113795> [Consulta: 24 de marzo de 2021]

GARCIA ARETIO, L. (2021). "COVID-19 y educación a distancia digital: preconfinamiento, confinamiento y posconfinamiento" en 5, (' ए 5HLMD ,EHRDP HUFDQD GH ( GXFDFlyQ D] ' LWDQFD, 24(1), pp. 09-32. $<$ http://dx.doi.org/10.5944/ried.24.1.28080> [Consulta: 09 de marzo de 2021]

GONZÁLEZ VIDEGARAY, M. del C. y Romero Ruiz, R. (2021). "La Cátedra Digital: un modelo hiflex para la

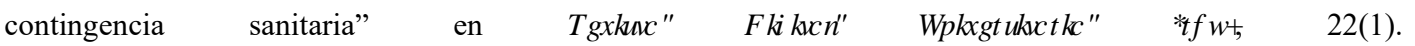
$<$ http://doi.org/10.22201/cuaieed.16076079e.2021.22.1.8> [Consulta: 08 de marzo de 2021]

INFORME COVID-19 CEPAL-UNESCO (2020). La educación en tiempos de la pandemia de COVID-19. $<$ https://repositorio.cepal.org/bitstream/handle/11362/45904/1/S2000510_es.pdf $>$ [Consulta: 09 de marzo de 2021]

LION, C. (2020). "Enseñar y aprender en tiempos de pandemia: presente y horizontes" en 6DEHHWI ISU FUFDVI5 HILWD $\begin{array}{llllll}G H & \text { ) IRRRItD } & \text { I } & \text { (GXFDFly } & 5(1), & 1-8 .\end{array}$ $<$ http://revistas.uncu.edu.ar/ojs3/index.php/saberesypracticas/article/view/3675> [Consulta: 12 de marzo de 2021]

MÁRQUEZ, C. (2018). "La emergencia de la universidad" en \&HMRQHM \%RJ GH SUFRDQ QUVII DFWDDOGDG $<$ https://marquezcarl.blogspot.com/p/lectio-quaestio-disputatio_28.html> [Consulta: 15 de marzo de 2021]

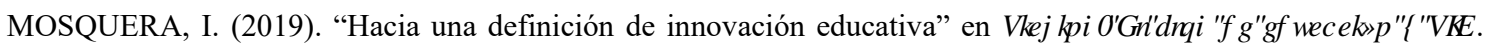
$<$ http://blog.tiching.com/definicion-innovacion-educativa/> [Consulta: 24 de marzo de 2021]

OPS ORGANIZACION PANAMERICANA DE LA SALUD (2020). ( QIHP HADCSRUHD\&RLRQDIUXVI\&2 9,' पिए. $<$ http://www.spain.info> [Consulta: 8 de marzo de 2021]

ORTEGA VÁZQUEZ, H.J. (2020). "Modelo instruccional idea. Una propuesta para el diseño de programas formativos en línea". 5HLLD \%ROHQQ 5HASH SRU 5HG ,EHRDP HUFDQD GH 3 HODJUtD, Vol. 9 (8), pp. 204-220. $<$ https://revista.redipe.org/index.php/1/article/view/1054> [Consulta: 17 de marzo de 2021]

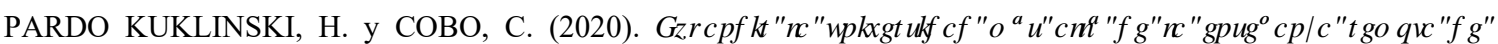
HP HJ HPFD, GHWKDFIDXQP RGHRIKEUURISRUASDQAP ID. Outliers School. Barcelona.

PINZÓN, G. (2021). "Pandemia y educación: Un cisne negro llamado COVID-19" en 9( 66凸( GX-RXLQDOODQJ XDUGD ( GXFDUMD, pp. 12-15. [Consulta: 16 de marzo de 2021]

PORLÁN, R. (2020). "El cambio de la enseñanza y el aprendizaje en tiempos de pandemia" en 5HLLDLGH( GXFDFIyQ $\begin{array}{llllll}\text { \$P EIHQWDO } & \text { II } & \text { 6RWHQIEIOCDO } & 2(1), & 1502 & \text { (2020). }\end{array}$ $<$ https://revistas.uca.es/index.php/REAyS/article/download/6168/6358/25926> [Consulta: 10 de marzo de 2021]

3 RUWD, QWWXFIRQDOGHD\&RGH IRI, QCRDP HUFDQRT6I\&] > https://www.indo.edu.mx/> [Consulta: 15 de marzo de 2021]

(cc) EY-NC-ND 2021, Universitat Politècnica de València

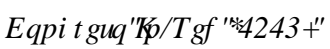


RIVAS, A. (2020). “Pedagogía de la excepción ¿cómo educar en la pandemia?”. Documento de trabajo en Universidad de San Andrés. [Consulta: 10 de marzo de 2021]

WALSH, K. (2020). "5 Reasons Students Today Need Innovative Learning Spaces” en ( PHJIQJ ( GHF $<$ https://www.emergingedtech.com/2020/04/reasons-students-need-innovative-learning-spaces/> [Consulta: 25 de marzo de 2021]

(cc) BY-NC-ND 2021, Universitat Politècnica de València

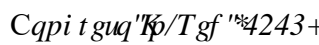

